\title{
Coupled quintic nonlinear Schrödinger equations in a generalized elastic solid
}

\author{
Irma Hacinliyan ${ }^{1}$ and Saadet Erbay ${ }^{2}$ \\ ${ }^{1}$ Department of Mathematics, Faculty of Science and Letters, Istanbul Technical University, \\ 34469 Maslak, Istanbul, Turkey \\ 2 Department of Mathematics, Faculty of Arts and Sciences, Isik University, 34398 Maslak, \\ Istanbul, Turkey \\ E-mail: hacinliy@itu.edu.tr and serbay@isikun.edu.tr
}

Received 4 May 2004, in final form 23 August 2004

Published 22 September 2004

Online at stacks.iop.org/JPhysA/37/9387

doi:10.1088/0305-4470/37/40/005

\begin{abstract}
In the present study, the nonlinear modulation of transverse waves propagating in a cubically nonlinear dispersive elastic medium is studied using a multiscale expansion of wave solutions. It is found that the propagation of quasimonochromatic transverse waves is described by a pair of coupled nonlinear Schrödinger (CNLS) equations. In the process of deriving the amplitude equations, it is observed that for a specific choice of material constants and wavenumber, the coefficient of nonlinear terms becomes zero, and the CNLS equations are no longer valid for describing the behaviour of transverse waves. In order to balance the nonlinear effects with the dispersive effects, by intensifying the nonlinearity, a new perturbation expansion is used near the critical wavenumber. It is found that the long time behaviour of the transverse waves about the critical wavenumber is given by a pair of coupled quintic nonlinear Schrödinger (CQNLS) equations. In the absence of one of the transverse waves, the CQNLS equations reduce to the single quintic nonlinear Schrödinger (QNLS) equation which has already been obtained in the context of water waves. By using a modified form of the so-called tanh method, some travelling wave solutions of the CQNLS equations are presented.
\end{abstract}

PACS numbers: 46.40.-f, 05.45.Yv 


\section{Introduction}

It is well known that the slowly varying amplitude of waves propagating as one-dimensional wave packets propagating in weakly nonlinear and weakly dispersive media is described by the single nonlinear Schrödinger (NLS) equation $[1,2]$

$$
\mathrm{i} u_{\tau}+p u_{\xi \xi}+q|u|^{2} u=0
$$

where $u$ is the complex amplitude, $\xi$ and $\tau$ are the slow space and time coordinates, respectively, $p$ and $q$ are real constants depending on material parameters, wave frequency and wavenumber. In certain cases, the coefficient of the nonlinear term, $q$, may vanish for a critical wavenumber, $k_{\mathrm{c}}$. It is clear that the NLS equation becomes inappropriate if $q\left(k_{\mathrm{c}}\right)=0$. The state where $p q=0$ is referred to as the marginal state of instability since the plane wave solutions of the single NLS equation are modulationally unstable (stable) if $p q>0(p q<0)$ when $q<0$. In the critical regime where $q\left(k_{\mathrm{c}}\right)=0$, dispersive and nonlinear effects do not balance each other, i.e., dispersion dominates over nonlinearity. In such a case, waves cannot propagate in the medium without changing their forms. In other words, solitary waves which imply perfect balance between nonlinearity and dispersion cannot exist in the medium. Thus to balance dispersive and nonlinear effects near the critical wavenumber, or the marginal state, higherorder terms must be considered in the evolution equation. Thus a new scaling is required to balance $u_{\tau}, u_{\xi \xi}$ and higher-order terms. This is accomplished either by re-scaling the time and distance or by intensifying the effects of nonlinearity. The modulation of Stokes waves near a critical wavenumber was first considered by Johnson [3]. In this study, the effect of dispersion was weakened by using slow scales $\epsilon^{4} t$ and $\epsilon^{2}\left(x-c_{\mathrm{g}} t\right)$ instead of $\epsilon^{2} t$ and $\epsilon\left(x-c_{\mathrm{g}} t\right)$, where $c_{\mathrm{g}}$ is the group speed of the transverse waves, and an evolution equation with higher-order nonlinear terms was derived

$$
\mathrm{i} u_{\tau}+p u_{\xi \xi}+r|u|^{4} u+\mathrm{i} a_{1}|u|^{2} u_{\xi}+\mathrm{i} a_{2} u\left(|u|^{2}\right)_{\xi}+a_{3} u \Psi_{\tau}=0
$$

with $\Psi_{\xi}=|u|^{2}$. In a later study [4], Kakutani and Michihiro also investigated the modulation of water waves near the critical wavenumber, i.e., the marginal state. They intensified the effect of nonlinearity by assuming that the nonlinearity is of $\mathcal{O}\left(\epsilon^{\frac{1}{2}}\right)$ instead of $\mathcal{O}(\epsilon)$, and they derived a governing equation for the complex amplitude $u$ near the critical wavenumber in the form

$$
\mathrm{i} u_{\tau}+p u_{\xi \xi}+r|u|^{4} u+\mathrm{i} a_{1}|u|^{2} u_{\xi}+\mathrm{i} a_{2} u\left(|u|^{2}\right)_{\xi}=0,
$$

which may be called the quintic nonlinear Schrödinger (QNLS) equation. Later, Parkes [5] derived formally the QNLS equation for a general system involving a single dependent variable. The same generalized nonlinear Schrödinger equation was also obtained in the study of pressure waves propagating in an infinitely long nonlinear elastic tube filled with an incompressible inviscid fluid [6]. As stated briefly above, the QNLS has found extensive application in water wave theory as well as other physical systems where the time and space scales are greater than those described by the cubic NLS equation. Because of this fact, various properties of this equation have been investigated in some detail [7-9].

If two waves co-propagate in weakly dispersive and nonlinear media, the slowly varying amplitudes of the two free waves are described with a pair of coupled nonlinear Schrödinger (CNLS) equations in various continuous media, such as optical fibres [10] and micro-polar elastic solids [11]

$$
\mathrm{i} u_{\tau}+p u_{\xi \xi}+q\left(|u|^{2}+|v|^{2}\right) u=0, \quad \mathrm{i} v_{\tau}+p v_{\xi \xi}+q\left(|u|^{2}+|v|^{2}\right) v=0,
$$

where $u$ and $v$ are the complex amplitudes of two free waves. The perturbation scheme leading to the CNLS equation assumes a balance between dispersive and nonlinear effects. However, 
in some cases the coefficient of nonlinear terms, $q$, in the CNLS equations (4) vanishes for a critical wavenumber as in the case of the single NLS equation. For such a case the CNLS system is no longer valid near the critical wavenumber. Thus, in order to balance nonlinear effects with dispersive effects, it is necessary to consider higher-order nonlinear terms for an appropriate description of wave packet solutions near the critical wavenumber. The main objective of the present study is to obtain a quintic coupled form of the single QNLS equation in the context of elasticity.

The outline of this paper is follows: the basic equations governing the displacement field of a cubically nonlinear elastic medium whose constitutive equation includes higher-order gradients are briefly given in section 2. Dispersion equations associated with the longitudinal and transverse components are also presented in the same section. Section 3 contains the derivation of evolution equations governing the nonlinear modulation of amplitudes of the transverse waves propagating in the above-mentioned infinite, homogeneous, weakly nonlinear and weakly dispersive elastic medium. Two independent complex amplitudes are required to describe the transverse components of the displacement field and as a consequence the modulation equations are found to be a pair of two coupled NLS equations. It is observed that the coefficient of the nonlinear terms of the coupled NLS equations vanishes at a critical wavenumber of the carrier wave. In this critical regime the dispersive and nonlinear effects do not balance each other, i.e., the coupled NLS equations are not appropriate. Near the critical wavenumber, a different ordering should be used to intensify the effects of nonlinearity. Thus, instead of assuming that the perturbed fields are of order $\epsilon$, they are assumed to be of order $\epsilon^{\frac{1}{2}}$. Then the resulting evolution equations describing the modulation of transverse waves near the critical wavenumber become a pair of coupled quintic nonlinear Schrödinger equations. As a special case, the coupled QNLS equations reduce to the single QNLS equation which has been already obtained for Stokes waves on the surface of water. Using a modified form of the so-called tanh method proposed by Yao and Li [12], some special solutions to the CQNLS equations are given in section 4 , and finally conclusions are presented.

\section{Basic equations and dispersion relations}

The present study considers transverse wave propagation near a critical wavenumber in a weakly nonlinear and weakly dispersive elastic medium, i.e., in a micromorphic elastic medium [13]. The motion of a material point in a micromorphic elastic solid is described by twelve scalar functions: three for the macro deformation of the material point and nine for the micro deformation of the micro elements in a macro volume. It is clear that the governing equations of such a generalized solid will become very complicated. However, assuming that the micro deformation of the micro elements are quite small, a great deal of simplifications have been made in the governing equations of the micromorphic elastic medium. Thus the resulting equations include higher-order spatial derivatives, representing the contribution of micro deformation, of three unknown functions, i.e., displacement components. The basic equations governing the displacement field of the cubically nonlinear elastic medium whose constitutive equations include higher-order displacement gradients have been given in [14]. The following set of equations describe the one-dimensional motion in the elastic medium:

$$
\begin{gathered}
u_{t t}-c_{\mathrm{L}}^{2} u_{x x}+4 c_{\mathrm{T}}^{2} m^{2}(1+v) u_{x x x x}=\gamma_{1} u_{x} u_{x x}+\gamma_{2}\left(v_{x} v_{x x}+w_{x} w_{x x}\right)+\gamma_{3}\left(u_{x}\right)^{2} u_{x x} \\
+\gamma_{4}\left\{u_{x x}\left[\left(v_{x}\right)^{2}+\left(w_{x}\right)^{2}\right]+2 u_{x}\left(v_{x} v_{x x}+w_{x} w_{x x}\right)\right\}, \\
v_{t t}-c_{\mathrm{T}}^{2} v_{x x}+4 c_{\mathrm{T}}^{2} m^{2} v_{x x x x}=\gamma_{2}\left(u_{x x} v_{x}+u_{x} v_{x x}\right)+\gamma_{4}\left[2 v_{x} u_{x} u_{x x}+\left(u_{x}\right)^{2} v_{x x}\right] \\
+\gamma_{5}\left[3\left(v_{x}\right)^{2} v_{x x}+2 v_{x} w_{x} w_{x x}+\left(w_{x}\right)^{2} v_{x x}\right],
\end{gathered}
$$




$$
\begin{aligned}
w_{t t}-c_{\mathrm{T}}^{2} w_{x x}+ & 4 c_{\mathrm{T}}^{2} m^{2} w_{x x x x}=\gamma_{2}\left(u_{x x} w_{x}+u_{x} w_{x x}\right)+\gamma_{4}\left[2 w_{x} u_{x} u_{x x}+\left(u_{x}\right)^{2} w_{x x}\right] \\
& +\gamma_{5}\left[3\left(w_{x}\right)^{2} w_{x x}+2 w_{x} v_{x} v_{x x}+\left(v_{x}\right)^{2} w_{x x}\right]
\end{aligned}
$$

where $t$ is time, $x$ is the coordinate in the direction of propagation, and $u$ is the longitudinal component whereas $v$ and $w$ are the transverse components of the displacement vector. The constants are defined as

$$
\begin{aligned}
& c_{\mathrm{L}}^{2}=(\lambda+2 \mu) / \rho_{0}, \quad c_{\mathrm{T}}^{2}=\mu / \rho_{0}, \\
& \gamma_{1}=[3(\lambda+2 \mu)+2(A+3 B+C)] / \rho_{0}, \\
& \gamma_{2}=(2 \lambda+4 \mu+A+2 B) / 2 \rho_{0}, \\
& \gamma_{3}=3[\lambda+2 \mu+4 A+12 B+4 C+8(D+G+H+K)] / 2 \rho_{0}, \\
& \gamma_{4}=(2 \lambda+4 \mu+5 A+14 B+4 C+6 G+4 H+8 K) / 4 \rho_{0}, \\
& \gamma_{5}=(\lambda+2 \mu+A+2 B+2 K) / 2 \rho_{0},
\end{aligned}
$$

where $c_{\mathrm{L}}$ and $c_{\mathrm{T}}$ are the speeds of longitudinal and transverse waves, respectively; $\lambda, \mu$ (Láme constants) are linear elastic constants, $A, B$ and $C$ are the second-order elastic constants, $D, G, H$ and $K$ are the third-order elastic constants, and $v$ and $m$ are new constants characterizing the microstructure. We should note that the one-dimensional governing equations (5) are obtained from a Hamiltonian principle

$$
\delta \iint \mathcal{L} \mathrm{d} x \mathrm{~d} t=0
$$

where $\mathcal{L}=\mathcal{T}-\Sigma$ is the Lagrangian, $\Sigma$ is the strain energy and $\mathcal{T}$ is the kinetic energy density functions. The Euler-Lagrange equations

$$
\frac{\partial}{\partial t}\left(\frac{\partial \mathcal{L}}{\partial u_{k, t}}\right)+\frac{\partial}{\partial x}\left(\frac{\partial \mathcal{L}}{\partial u_{k, x}}\right)-\frac{\partial^{2}}{\partial x^{2}}\left(\frac{\partial \mathcal{L}}{\partial u_{k, x x}}\right)=0, \quad(k=1,2,3)
$$

lead to governing equations (5) that involve cubic nonlinear terms, where $\left(u_{1}, u_{2}, u_{3}\right)=$ $(u, v, w)$. This is due to the fact that we have considered a third-order elastic material for which the strain energy density function $\Sigma$ was assumed to be a fourth-degree polynomial of displacement gradients $u_{k, l}[14]$.

The dispersion relations obtained from the linearized equations of system (5) are given in the form

$$
\begin{aligned}
& D_{1}(k, \omega)=\omega^{2}-c_{\mathrm{L}}^{2} k^{2}-4 c_{\mathrm{T}}^{2} m^{2}(1+v) k^{4}, \\
& D_{2}(k, \omega)=D_{3}(k, \omega)=\omega^{2}-c_{\mathrm{T}}^{2} k^{2}-4 c_{\mathrm{T}}^{2} m^{2} k^{4}
\end{aligned}
$$

where $k$ and $\omega$ denote wavenumber and frequency, respectively. In equation (6), $D_{1}$ represents the dispersion relation corresponding to the longitudinal displacement mode associated with $u$, and $D_{2}$ and $D_{3}$ represent the dispersion relations corresponding to the transverse displacement modes associated with $v$ and $w$, respectively. As can be seen from equations (6), both the longitudinal mode and the transverse modes are dispersive. Since the transverse modes satisfy the same dispersion relation, one may expect a wave-wave interaction between the transverse modes if the nonlinearity is included in the analysis.

\section{Wave modulation near a critical wavenumber}

In recent years, wave propagation problems have drawn a great attention in the context of elasticity. In particular, the interaction between longitudinal and transverse wave envelopes has been addressed in a number of works [15-17]. In the present section, we are concerned with the interaction of two transverse waves propagating in an infinite elastic medium. 
In particular, the self-modulation of two transverse waves in a weakly nonlinear and weakly dispersive elastic medium will be considered, and the contribution of higher-order nonlinear effects on the wave propagation near a critical wavenumber is investigated. The modulation of quasi-monochromatic waves will be studied using an asymptotic method which requires the expansion of field variables in terms of a small amplitude parameter $\epsilon$ and introduces the stretched coordinates [18].

The modulation of transverse waves propagating in a cubically nonlinear and dispersive elastic medium has been considered in a recent study [19] using a multi-scale perturbation method. In this study, the following stretched coordinates are introduced:

$$
\xi=\epsilon\left(x-c_{\mathrm{g}} t\right), \quad \tau=\epsilon^{2} t,
$$

where $\xi$ and $\tau$ are the slow variables, and $c_{\mathrm{g}}$ the group speed of the transverse waves, and the field variables, the components of the displacement vector, are expressed as power series of the small parameter $\epsilon$

$$
\begin{aligned}
& u=\epsilon u_{1}^{(0)}(\xi, \tau)+\epsilon^{2}\left[u_{2}^{(0)}(\xi, \tau)+u_{2}^{(2)}(\xi, \tau) \mathrm{e}^{2 \mathrm{i} \theta}+\text { c.c. }\right]+\cdots \\
& v=\epsilon\left[\phi(\xi, \tau) \mathrm{e}^{\mathrm{i} \theta}+\text { c.c. }\right]+\epsilon^{2}\left[v_{2}^{(0)}(\xi, \tau)+v_{2}^{(1)}(\xi, \tau) \mathrm{e}^{\mathrm{i} \theta}+v_{2}^{(2)}(\xi, \tau) \mathrm{e}^{2 \mathrm{i} \theta}+\text { c.c. }\right]+\cdots \\
& w=\epsilon\left[\psi(\xi, \tau) \mathrm{e}^{\mathrm{i} \theta}+\text { c.c. }\right]+\epsilon^{2}\left[w_{2}^{(0)}(\xi, \tau)+w_{2}^{(1)}(\xi, \tau) \mathrm{e}^{\mathrm{i} \theta}+w_{2}^{(2)}(\xi, \tau) \mathrm{e}^{2 \mathrm{i} \theta}+\text { c.c. }\right]+\cdots
\end{aligned}
$$

where $\phi$ and $\psi$ are the free first-order transverse displacement components, $\theta=k x-\omega t$ and c.c. stands for the complex conjugate of the preceding terms. Substituting solutions (8) together with the stretched coordinates (7) into the governing equations (5), a hierarchy of equations in terms of powers of $\epsilon$ is obtained. Solutions of the perturbation equations lead to the compatibility conditions in the form of a pair of two coupled nonlinear Schödinger equations [19]

$$
\begin{aligned}
& \mathrm{i} \phi_{\tau}+p \phi_{\xi \xi}+\delta_{1}|\phi|^{2} \phi+\delta_{2} \psi^{2} \phi^{*}+\delta_{3}|\psi|^{2} \phi=0, \\
& \mathrm{i} \psi_{\tau}+p \psi_{\xi \xi}+\delta_{1}|\psi|^{2} \psi+\delta_{2} \phi^{2} \psi^{*}+\delta_{3}|\phi|^{2} \psi=0,
\end{aligned}
$$

where

$$
\begin{array}{ll}
p=-\frac{1}{2 \omega}\left(c_{\mathrm{g}}^{2}-c_{\mathrm{T}}^{2}-24 c_{\mathrm{T}}^{2} m^{2} k^{2}\right), & \delta_{1}=\delta_{2}+\delta_{3}, \\
\delta_{2}=-\frac{\gamma_{2}^{2} k^{6}}{\omega D_{1}(2 k, 2 \omega)}-\frac{\gamma_{5} k^{4}}{2 \omega}, & \delta_{3}=-\frac{\gamma_{2}^{2} k^{4}}{2 \omega\left(c_{\mathrm{g}}^{2}-c_{\mathrm{L}}^{2}\right)}-\frac{\gamma_{5} k^{4}}{\omega} .
\end{array}
$$

The CNLS equations (9) reduce to the Manakov system if the coefficient $\delta_{2}=0$

$\mathrm{i} \phi_{\tau}+p \phi_{\xi \xi}+\delta\left(|\phi|^{2}+|\psi|^{2}\right) \phi=0, \quad \mathrm{i} \psi_{\tau}+p \psi_{\xi \xi}+\delta\left(|\psi|^{2}+|\phi|^{2}\right) \psi=0$,

where

$$
\delta_{1}=\delta_{3}=\delta=\frac{\gamma_{2}^{2} k^{4}}{2 \omega}\left[\frac{4 k^{2}}{D_{1}(2 k, 2 \omega)}-\frac{1}{c_{\mathrm{g}}^{2}-c_{\mathrm{L}}^{2}}\right] .
$$

It should be noted that the assumption $\delta_{2}=0$ imposes a restriction on the value of the wavenumber $k$, i.e. $\gamma_{5}=-2 \gamma_{2}^{2} k^{2} / D_{1}(2 k, 2 \omega)$. The Manakov system (10) is derived by balancing the effects of weak nonlinearity and small side-band width. In the course of derivation, the nonlinearity and small-side band effects are assumed to be of $\mathcal{O}(\epsilon)$ implying that the dispersive and nonlinear coefficients of the CNLS equations are of $\mathcal{O}(1)$. However, for certain cases i.e., when the wavenumber $k$ is near a critical wavenumber $k_{\mathrm{c}}$, the nonlinear term $\delta$ may become zero. As is seen from equations (6) and (11), $\delta$ becomes zero when the wavenumber is given by

$$
k_{\mathrm{c}}^{2}=-\frac{3+2 v}{2 m^{2}(7+4 v)}
$$


with $-7<4 v<-6$. Condition (12) together with $\gamma_{5}=-2 \gamma_{2}^{2} k^{2} / D_{1}(2 k, 2 \omega)$ gives rise to

$$
\frac{\gamma_{5}}{\gamma_{2}^{2}}=-\frac{(7+4 v)}{2\left(c_{\mathrm{T}}^{2}-c_{\mathrm{L}}^{2}\right)(7+4 v)+4 c_{\mathrm{T}}^{2}(3+2 v)(3+4 v)} \text {. }
$$

Equation (13) gives a relation between linear, second-order and third-order elastic constants and microstructure constant. Since the wavenumber of a transverse wave can be chosen arbitrarily, the critical wavenumber $k_{\mathrm{c}}$ exists provided that condition (13) is satisfied. Under the assumption of existence of such a critical wavenumber $k_{\mathrm{c}}$, similar to the marginal state of the single NLS equation, the nonlinear terms drop from system (10) and the CNLS equations cannot be valid about $k_{\mathrm{c}}$. In such a case, new evolution equations are needed to describe the evolution of transverse modes near the critical wavenumber. Thus, a new scaling must be used to balance the nonlinear and dispersive effects. To this end, the effect of nonlinearity is intensified in the perturbation expansion, and nonlinearity is assumed to be of $\mathcal{O}\left(\epsilon^{\frac{1}{2}}\right)$ instead of $\mathcal{O}(\epsilon)$. In other words, solutions to the system (5) are expanded into half powers of $\epsilon$ to include the missing nonlinear terms into the coupled evolution equations near the critical wavenumber $k_{\mathrm{c}}$ (for the sake of simplicity the subscript $c$ will be omitted in the rest of the work):

$$
\begin{aligned}
& u=u_{1}^{(0)}(\xi, \tau)+\epsilon\left[u_{2}^{(0)}(\xi, \tau)+u_{2}^{(2)}(\xi, \tau) \mathrm{e}^{2 \mathrm{i} \theta}+\text { c.c. }\right]+\epsilon^{\frac{3}{2}} u_{3}^{(0)}(\xi, \tau) \\
& +\epsilon^{2}\left[u_{4}^{(0)}(\xi, \tau)+u_{4}^{(2)}(\xi, \tau) \mathrm{e}^{2 \mathrm{i} \theta}+u_{4}^{(4)}(\xi, \tau) \mathrm{e}^{4 \mathrm{i} \theta}+\text { c.c. }\right]+\cdots \\
& v=\epsilon^{\frac{1}{2}}\left[\phi(\xi, \tau) \mathrm{e}^{\mathrm{i} \theta}+\mathrm{c} . \mathrm{c}\right]+\epsilon v_{2}^{(0)}(\xi, \tau)+\epsilon^{\frac{3}{2}} v_{3}^{(0)}(\xi, \tau)+\epsilon^{2} v_{4}^{(0)}(\xi, \tau)+\cdots \\
& w=\epsilon^{\frac{1}{2}}\left[\psi(\xi, \tau) \mathrm{e}^{\mathrm{i} \theta}+\text { c.c }\right]+\epsilon w_{2}^{(0)}(\xi, \tau)+\epsilon^{\frac{3}{2}} w_{3}^{(0)}(\xi, \tau)+\epsilon^{2} w_{4}^{(0)}(\xi, \tau)+\cdots .
\end{aligned}
$$

Note that the free transverse modes $\phi$ and $\psi$ are of $\mathcal{O}\left(\epsilon^{\frac{1}{2}}\right)$ instead of $\mathcal{O}(\epsilon)$. Here we should note that, due to intensified nonlinear effects near the critical wavenumber, we have to consider higher order terms in governing equations (5) as well as in expansion (14). In order to have higher-order nonlinear terms in the governing equations, fifth-order and sixth-order displacement gradients must be included in the strain energy density function $\Sigma$ as discussed in section 2. In such a case, the strain energy density function $\Sigma$, and consequently the governing equations will have very complicated forms. Thus the computation of the coefficients in the evolution equations will be complicated too. On the other hand, even if we do consider higherorder terms, we would not be able to know the contribution of higher-order elastic constants in the coefficients since their values are not known. Therefore we have omitted higher-order nonlinear terms in the governing equations.

Substituting solutions (14) together with the stretched coordinates (7) into the governing equations (5), we obtain a hierarchy of equations in terms of half powers of $\epsilon$. For $\mathcal{O}\left(\epsilon^{\frac{1}{2}}\right)$, using the fact that $D_{2}(k, \omega)=0$, we find that the transverse modes $\phi$ and $\psi$ are arbitrary functions:

$$
\mathrm{e}^{\mathrm{i} \theta}: D_{2}(k, \omega) \phi=0, \quad D_{2}(k, \omega) \psi=0 .
$$

For $\mathcal{O}(\epsilon)$, setting the coefficients of the second modes equal to zero, we express the second-order longitudinal mode in terms of the first-order transverse modes

$$
\mathrm{e}^{2 \mathrm{i} \theta}: u_{2}^{(2)}=\frac{\mathrm{i} k^{3} \gamma_{2}}{D_{1}(2 k, 2 \omega)}\left(\phi^{2}+\psi^{2}\right)
$$

Substituting expression (16) for $u_{2}^{(2)}$ into the perturbation equations obtained for $\mathcal{O}\left(\epsilon^{\frac{3}{2}}\right)$, and then comparing terms of the same modes, we obtain the following expressions:

$$
\begin{aligned}
& \mathrm{e}^{\mathrm{i} \theta}: D_{1}(2 k, 2 \omega)\left(-2 \mathrm{i} k \phi^{*} u_{2}^{(2)}+\phi u_{1, \xi}^{(0)}\right)-2 \gamma_{2} k^{4}\left(3|\phi|^{2} \phi+\phi^{*} \psi^{2}+2|\psi|^{2} \phi\right)=0, \\
& D_{1}(2 k, 2 \omega)\left(-2 \mathrm{i} k \psi^{*} u_{2}^{(2)}+\psi u_{1, \xi}^{(0)}\right)-2 \gamma_{2} k^{4}\left(3|\psi|^{2} \psi+\psi^{*} \phi^{2}+2|\phi|^{2} \psi\right)=0 .
\end{aligned}
$$


In order to obtain explicit forms of these expressions, we need to know the explicit form of $u_{1, \xi}^{(0)}$. Thus, we proceed to the next-order problem. In $\mathcal{O}\left(\epsilon^{2}\right)$, we obtain the following:

$\mathrm{e}^{\mathrm{i} 0}: u_{1, \xi}^{(0)}=\frac{k^{2} \gamma_{2}}{c_{\mathrm{g}}^{2}-c_{\mathrm{L}}^{2}}\left(|\phi|^{2}+|\psi|^{2}\right)$,

$\mathrm{e}^{2 \mathrm{i} \theta}: D_{1}(2 k, 2 \omega) u_{4}^{(2)}=4 k^{2} \gamma_{1} u_{1, \xi}^{(0)} u_{2}^{(2)}+3 k^{2} \gamma_{2}\left(\phi \phi_{\xi}+\psi \psi_{\xi}\right)$

$+2 \gamma_{4} k^{3}\left[4 k u_{2}^{(2)}\left(|\phi|^{2}+|\psi|^{2}\right)+\mathrm{i} u_{1, \xi}^{(0)}\left(\phi^{2}+\psi^{2}\right)\right]$

$\mathrm{e}^{4 \mathrm{i} \theta}: D_{1}(4 k, 4 \omega) u_{4}^{(4)}=\frac{-8 \mathrm{i} k^{7} \gamma_{2}}{D_{1}^{2}(2 k, 2 \omega)}\left(k^{2} \gamma_{1} \gamma_{2}+\gamma_{4} D_{1}(2 k, 2 \omega)\right)\left(\phi^{2}+\psi^{2}\right)^{2}$.

In order to eliminate the self-resonance we require that the dispersion relation $D_{2}(n k, n \omega)$ is not satisfied by the pairs $(n k, n \omega)$ for $n \geqslant 2$. Therefore the second-order longitudinal mode is expressed by the lower order transverse modes:

$$
u_{4}^{(2)}=a_{1}\left(\phi \phi_{\xi}+\psi \psi_{\xi}\right)+\mathrm{i} a_{2}\left(|\phi|^{2}+|\psi|^{2}\right)\left(\phi^{2}+\psi^{2}\right)
$$

where

$$
\begin{aligned}
& a_{1}=\frac{\gamma_{2} k^{2}}{D_{1}^{2}(2 k, 2 \omega)}\left\{3 D_{1}(2 k, 2 \omega)-8 k\left[\omega c_{\mathrm{g}}-c_{\mathrm{L}}^{2} k-32 c_{\mathrm{T}}^{2} m^{2}(1+v) k^{3}\right]\right\}, \\
& a_{2}=\frac{4 \gamma_{2} k^{5}}{\left(c_{\mathrm{g}}^{2}-c_{\mathrm{L}}^{2}\right)^{2} D_{1}^{2}(2 k, 2 \omega)}\left[\gamma_{4}\left(c_{\mathrm{g}}^{2}-c_{\mathrm{L}}^{2}\right) D_{1}(2 k, 2 \omega)+\gamma_{1} \gamma_{2} k^{2}\right] .
\end{aligned}
$$

Since we do not need functions obtained from higher-order modes of $\mathcal{O}\left(\epsilon^{2}\right)$ their explicit forms will not be given here.

After having found the explicit form of the function $u_{1, \xi}^{(0)}$, we find from (17) that

$$
\delta \phi\left(|\phi|^{2}+|\psi|^{2}\right)=0, \quad \delta \psi\left(|\phi|^{2}+|\psi|^{2}\right)=0
$$

where $\delta$ is the coefficient of the cubic nonlinear terms of the CNLS equations, i.e. (20) gives an identity.

For the first mode of $\mathcal{O}\left(\epsilon^{\frac{5}{2}}\right)$, we obtain the two coupled equations for the transverse modes

$$
\begin{aligned}
\mathrm{e}^{\mathrm{i} \theta}: 2 \mathrm{i} \omega \phi_{\tau}- & \left(c_{\mathrm{g}}^{2}-c_{\mathrm{T}}^{2}-24 c_{\mathrm{T}}^{2} m^{2} k^{2}\right) \phi_{\xi \xi}=\gamma_{2} k\left[k\left(\phi u_{2, \xi}^{(0)}-3 \phi^{*} u_{2, \xi}^{(2)}\right)-\mathrm{i}\left(\phi u_{1, \xi \xi}^{(0)}+2 \phi_{\xi} u_{1, \xi}^{(0)}\right.\right. \\
& \left.\left.+2 k^{2} \phi^{*} u_{4}^{(2)}\right)\right]+\gamma_{4} k^{2}\left[\left(u_{1, \xi}^{(0)}\right)^{2} \phi+8 k^{2}\left|u_{2}^{(2)}\right|^{2} \phi-4 \mathrm{i} k u_{1, \xi}^{(0)} u_{2}^{(2)} \phi^{*}\right] \\
& -4 \mathrm{i} \gamma_{5} k^{3}\left[3|\phi|^{2} \phi_{\xi}+|\psi|^{2} \phi_{\xi}+\phi^{*} \psi \psi_{\xi}+\phi \psi^{*} \psi_{\xi}\right] \\
2 \mathrm{i} \omega \psi_{\tau}-\left(c_{\mathrm{g}}^{2}-\right. & \left.c_{\mathrm{T}}^{2}-24 c_{\mathrm{T}}^{2} m^{2} k^{2}\right) \psi_{\xi \xi}=\gamma_{2} k\left[k\left(\psi u_{2, \xi}^{(0)}-3 \psi^{*} u_{2, \xi}^{(2)}\right)-\mathrm{i}\left(\psi u_{1, \xi \xi}^{(0)}+2 \psi_{\xi} u_{1, \xi}^{(0)}\right.\right. \\
& \left.\left.+2 k^{2} \psi^{*} u_{4}^{(2)}\right)\right]+\gamma_{4} k^{2}\left[\left(u_{1, \xi}^{(0)}\right)^{2} \psi+8 k^{2}\left|u_{2}^{(2)}\right|^{2} \psi-4 \mathrm{i} k u_{1, \xi}^{(0)} u_{2}^{(2)} \psi^{*}\right] \\
& -4 \mathrm{i} \gamma_{5} k^{3}\left[3|\psi|^{2} \psi_{\xi}+|\phi|^{2} \psi_{\xi}+\psi^{*} \phi \phi_{\xi}+\psi \phi^{*} \psi_{\xi}\right] .
\end{aligned}
$$

Here $u_{1, \xi}^{(0)}$ and $u_{2}^{(2)}$ are expressions known explicitly in terms of the first-order quantities. In order to express the coupled evolution equations in a compact form, all the higher-order longitudinal modes should be written in the system. In this regard we should calculate $u_{2, \xi}^{(0)}$ from the higher-order perturbation equations. From the zeroth mode of $\mathcal{O}\left(\epsilon^{3}\right)$ we have

$$
\begin{aligned}
\left(c_{\mathrm{g}}^{2}-c_{\mathrm{L}}^{2}\right) u_{2, \xi \xi}^{(0)} & -2 c_{\mathrm{g}} u_{1, \tau \xi}^{(0)}=\frac{\gamma_{1}}{2}\left[\left(u_{1, \xi}^{(0)}\right)^{2}+8 k^{2}\left|u_{2}^{(2)}\right|^{2}\right]_{\xi}+\mathrm{i} \gamma_{2} k\left[\phi \phi_{\xi}^{*}-\phi^{*} \phi_{\xi}+\psi \psi_{\xi}^{*}-\psi^{*} \psi_{\xi}\right]_{\xi} \\
& +2 \gamma_{4} k^{2}\left\{u_{1, \xi}^{(0)}\left(|\phi|^{2}+|\psi|^{2}\right)-\mathrm{i} k u_{2}^{(2)}\left[\left(\phi^{*}\right)^{2}+\left(\psi^{*}\right)^{2}\right]+\mathrm{i} k u_{2}^{(2) *}\left(\phi^{2}+\psi^{2}\right)\right\}_{\xi}
\end{aligned}
$$

Integrating equation (22) with respect to $\xi$ once, and substituting expressions (16) for $u_{2}^{(2)}$ and

$$
u_{1, \tau}^{(0)}=\frac{k^{2} \gamma_{2}}{c_{\mathrm{g}}^{2}-c_{\mathrm{L}}^{2}} \int^{\xi} \frac{\partial}{\partial \tau}\left(|\phi|^{2}+|\psi|^{2}\right) \mathrm{d} \zeta,
$$


into (22), we obtain

$$
\begin{aligned}
\left(c_{\mathrm{g}}^{2}-c_{\mathrm{L}}^{2}\right) u_{2, \xi}^{(0)} & =\mathrm{i} \gamma_{2} k\left(\phi \phi_{\xi}^{*}-\phi^{*} \phi_{\xi}+\psi \psi_{\xi}^{*}-\psi^{*} \psi_{\xi}\right) \\
& +\frac{\gamma_{2} k^{4}}{2\left(c_{\mathrm{g}}^{2}-c_{\mathrm{L}}^{2}\right)^{2}}\left[4 \gamma_{4}\left(c_{\mathrm{g}}^{2}-c_{\mathrm{L}}^{2}\right)+\gamma_{1} \gamma_{2}\right]\left(|\phi|^{2}+|\psi|^{2}\right)^{2} \\
& +\frac{4 \gamma_{2} k^{6}}{D_{1}^{2}(2 k, 2 \omega)}\left[\gamma_{4} D_{1}(2 k, 2 \omega)-\gamma_{1} \gamma_{2} k^{2}\right]\left|\phi^{2}+\psi^{2}\right|^{2} \\
& +\frac{2 c_{\mathrm{g}} k^{2} \gamma_{2}}{c_{\mathrm{g}}^{2}-c_{\mathrm{L}}^{2}} \int^{\xi} \frac{\partial}{\partial \tau}\left(|\phi|^{2}+|\psi|^{2}\right) \mathrm{d} \zeta,
\end{aligned}
$$

where integration is performed over the interval $(-\infty, \xi)$. Substituting the previously obtained functions $u_{1, \xi}^{(0)}, u_{2, \xi}^{(0)}$ and $u_{4}^{(2)}$ in the coupled evolution equations (21), after tedious but straightforward calculations we obtain the following two coupled equations for the transverse modes:

$$
\begin{array}{rl}
\mathrm{i} \phi_{\tau}+p \phi_{\xi \xi}+\mathrm{i} & a \phi^{*}\left(\phi \phi_{\xi}+\psi \psi_{\xi}\right)+5 b \phi|\phi|^{4}+2 b \phi^{*} \psi^{2}|\psi|^{2}+6 b \phi|\phi|^{2}|\psi|^{2} \\
+ & 3 b \phi^{*} \psi^{2}|\phi|^{2}+b \phi^{3}\left(\psi^{*}\right)^{2}+3 b \phi|\psi|^{4}+c \phi \int^{\xi} \frac{\partial}{\partial \tau}\left(|\phi|^{2}+|\psi|^{2}\right) \mathrm{d} \zeta=0 \\
\mathrm{i} \psi_{\tau}+p \psi_{\xi \xi}+\mathrm{i} a \psi^{*}\left(\psi \psi_{\xi}+\phi \phi_{\xi}\right)+5 b \psi|\psi|^{4}+2 b \psi^{*} \phi^{2}|\phi|^{2}+6 b \psi|\psi|^{2}|\phi|^{2} \\
+3 b \psi^{*} \phi^{2}|\psi|^{2}+b \psi^{3}\left(\phi^{*}\right)^{2}+3 b \psi|\phi|^{4}+c \psi \int^{\xi} \frac{\partial}{\partial \tau}\left(|\psi|^{2}+|\phi|^{2}\right) \mathrm{d} \zeta=0
\end{array}
$$

where the coefficient $p$ is given in (11)

$$
\begin{aligned}
& a=-\frac{4 \gamma_{2}^{2} k^{5}}{2 \omega D_{1}^{2}(2 k, 2 \omega)}\left[D_{1}(2 k, 2 \omega)-4 k\left(\omega c_{\mathrm{g}}-c_{\mathrm{L}}^{2} k-32 c_{\mathrm{T}}^{2} m^{2}(1+v) k^{3}\right)\right], \\
& b=-\frac{8 \gamma_{2}^{2} k^{10}}{D_{1}^{3}(2 k, 2 \omega)}\left(3 \gamma_{4} D_{1}(2 k, 2 \omega)+2 \gamma_{1} \gamma_{2} k^{2}\right), \quad c=-\frac{2 \gamma_{2} k^{2}}{\left(c_{\mathrm{g}}^{2}-c_{\mathrm{L}}^{2}\right)} .
\end{aligned}
$$

The coupled integro-differential equations (24) describe the motion of the two linearly polarized transverse waves, polarized along the $y$ and $z$ directions, respectively, about a critical wavenumber. Note that the coupled nonlinear terms in system (24) are very complicated. A straightforward but tedious algebra shows that it is not possible to remove the integral terms from system (24). At this stage, both, to simplify the highly complicated nonlinear terms and to remove the integral terms in (24) we rewrite the coupled evolution equations (24) in terms of the circularly polarized waves defined as

$$
u^{\prime}=\frac{1}{\sqrt{2}}(\phi-\mathrm{i} \psi), \quad v^{\prime}=\frac{1}{\sqrt{2}}(\phi+\mathrm{i} \psi)
$$

where $|\phi|^{2}+|\psi|^{2}=\left|u^{\prime}\right|^{2}+\left|v^{\prime}\right|^{2}$. Thus the system takes the following form in terms of the circularly polarized transverse waves (after dropping primes)

$$
\begin{aligned}
& \mathrm{i} u_{\tau}+p u_{\xi \xi}+\mathrm{i} a(u v)_{\xi} v^{*}+2 b\left(|u|^{4}+6|u|^{2}|v|^{2}+3|v|^{4}\right) u+c u \int^{\xi} \frac{\partial}{\partial \tau}\left(|u|^{2}+|v|^{2}\right) \mathrm{d} \zeta=0 \\
& \mathrm{i} v_{\tau}+p v_{\xi \xi}+\mathrm{i} a(u v)_{\xi} u^{*}+2 b\left(|v|^{4}+6|u|^{2}|v|^{2}+3|u|^{4}\right) v+c v \int^{\xi} \frac{\partial}{\partial \tau}\left(|v|^{2}+|u|^{2}\right) \mathrm{d} \zeta=0
\end{aligned}
$$

We note that the coupled nonlinear terms in (26) have a more simplified form than that of (24). In order to eliminate the integral terms from system (26), we multiply the first equation of (26) by $u^{*}$ and subtract from the complex conjugate of the same equation. The result is

$$
\mathrm{i}\left(|u|^{2}\right)_{\tau}=-p\left(u^{*} u_{\xi}-u u_{\xi}^{*}\right)_{\xi}-\mathrm{i} a\left(|v|^{2}|u|^{2}\right)_{\xi} .
$$


Similarly, we obtain

$$
\mathrm{i}\left(|v|^{2}\right)_{\tau}=-p\left(v^{*} v_{\xi}-v v_{\xi}^{*}\right)_{\xi}-\mathrm{i} a\left(|v|^{2}|u|^{2}\right)_{\xi}
$$

by performing similar computations for $(26)_{2}$. We note that (27) and (28) are mass conservation laws associated with $u$ and $v$ components, respectively. Thus the sum of (27) and (28) leads to

$$
\int^{\xi} \frac{\partial}{\partial \tau}\left(|u|^{2}+|v|^{2}\right) \mathrm{d} \zeta=\mathrm{i} p\left(u^{*} u_{\xi}-u u_{\xi}^{*}+v^{*} v_{\xi}-v v_{\xi}^{*}\right)-2 a|u|^{2}|v|^{2},
$$

where the solutions $u$ and $v$ are rapidly decreasing functions of $\xi$ as $\xi \rightarrow-\infty$. Using the result (29) in the coupled integro-differential equations (26) gives the following set of equations:

$$
\begin{aligned}
\mathrm{i} u_{\tau}+p u_{\xi \xi}+\mathrm{i}[ & \left.p c\left(u^{*} u_{\xi}-u u_{\xi}^{*}+v^{*} v_{\xi}-v v_{\xi}^{*}\right) u+a(u v)_{\xi} v^{*}\right]+\left[2 b|u|^{4}\right. \\
& \left.+(12 b-2 a c)|u|^{2}|v|^{2}+6 b|v|^{4}\right] u=0, \\
\mathrm{i} v_{\tau}+p v_{\xi \xi}++\mathrm{i}\left[p c\left(v^{*} v_{\xi}-v v_{\xi}^{*}+u^{*} u_{\xi}-u u_{\xi}^{*}\right) v+a(u v)_{\xi} u^{*}\right]+\left[2 b|v|^{4}\right. & \left.+(12 b-2 a c)|v|^{2}|u|^{2}+6 b|u|^{4}\right] v=0 .
\end{aligned}
$$

The coupled evolution equations (30) with quintic nonlinear terms describe the propagation of transverse waves near the critical wavenumber, $k_{\mathrm{c}}$. System (30) reduces to the single quintic nonlinear Schrödinger equation obtained previously in the context of water waves if one of the components is assumed to be identically zero, i.e. $v \equiv 0$

$$
\mathrm{i} u_{\tau}+p u_{\xi \xi}+\mathrm{i} p c\left[2|u|^{2} u_{\xi}-u\left(|u|^{2}\right)_{\xi}\right]+2 b|u|^{4} u=0 .
$$

In addition, the coupled system (30) contains a derivative nonlinear Schrödinger equation as a spacial case when $v=0$ and the coefficient of the quintic nonlinear term $b=0$.

Similarly, if solutions with $u \rightarrow u_{0}$ and $v \rightarrow v_{0}, u^{\prime}, v^{\prime}, \ldots, \rightarrow 0$ are allowed as $\xi \rightarrow-\infty$ then the sum of (27) and (28) gives

$$
\int^{\xi} \frac{\partial}{\partial \tau}\left(|u|^{2}+|v|^{2}\right) \mathrm{d} \zeta=\mathrm{i} p\left(u^{*} u_{\xi}-u u_{\xi}^{*}+v^{*} v_{\xi}-v v_{\xi}^{*}\right)-2 a|u|^{2}|v|^{2}+2 a\left|u_{0}\right|^{2}\left|v_{0}\right|^{2} .
$$

If the result (32) is substituted into the coupled integro-differential equations (26), we obtain the following set of equations:

$$
\begin{aligned}
& \mathrm{i} u_{\tau}+p u_{\xi \xi}+\mathrm{i}[\left.p c\left(u^{*} u_{\xi}-u u_{\xi}^{*}+v^{*} v_{\xi}-v v_{\xi}^{*}\right) u+a(u v)_{\xi} v^{*}\right] \\
&+\left[2 b|u|^{4}+(12 b-2 a c)|u|^{2}|v|^{2}+6 b|v|^{4}\right] u+2 a c\left|u_{0}\right|^{2}\left|v_{0}\right|^{2} u=0 \\
& \mathrm{i} v_{\tau}+p v_{\xi \xi}+\mathrm{i}[\left.p c\left(v^{*} v_{\xi}-v v_{\xi}^{*}+u^{*} u_{\xi}-u u_{\xi}^{*}\right) v+a(u v)_{\xi} u^{*}\right] \\
&+\left[2 b|v|^{4}+(12 b-2 a c)|v|^{2}|u|^{2}+6 b|u|^{4}\right] v+2 a c\left|u_{0}\right|^{2}\left|v_{0}\right|^{2} v=0 .
\end{aligned}
$$

The coupled evolution equations (33), in a similar fashion, reduces to a single quintic nonlinear Schrödinger equation, if $v \equiv 0$

$$
\mathrm{i} u_{\tau}+p u_{\xi \xi}+\mathrm{i} p c\left[2|u|^{2} u_{\xi}-u\left(|u|^{2}\right)_{\xi}\right]+2 b|u|^{4} u=0 .
$$

Similar to that of (30), the coupled system (33) contains a derivative nonlinear Schrödinger equation as a spacial case when $v=0$ and the coefficient $b=0$. Various one-component forms of cubic, quintic and derivative Schrödinger equations in nonlinear optics, plasma physics and fluid dynamics have also been listed in [8] and references therein. In conclusion, both of the systems (30) and (33) may be called the coupled quintic nonlinear Schrödinger equations. 


\section{Some special solutions to CQNLS equations}

In recent years, some special solutions to a single QNLS equation which is a one-component form of the CQNLS system have been obtained in [8, 9] using different approaches. In [8], Pathria and Morris have obtained oscillatory, phase jump and solitary wave solution to a single QNLS equation by transforming the equation to one from which exact solutions are found. Clarkson has obtained dimensional reductions and exact solutions of a QNLS equation via a direct algorithmic method that does not use group theoretical approach [9]. In another study [22], Fedele has used a correspondence between a generalized Korteweg-de Vries equation and a generalized nonlinear Schrödinger equation to find a positive stationary-profile solution to a quintic NLS equation. In the present section, we consider a special form of the CQNLS equations (30)

$$
\begin{aligned}
& \mathrm{i} u_{\tau}+q_{1} u_{\xi \xi}+\mathrm{i} q_{2}\left(u^{*} u_{\xi}-u u_{\xi}^{*}+v^{*} v_{\xi}-v v_{\xi}^{*}\right) u+q_{3}\left(|u|^{4}+6|u|^{2}|v|^{2}+3|v|^{4}\right) u=0, \\
& \mathrm{i} v_{\tau}+q_{1} v_{\xi \xi}+\mathrm{i} q_{2}\left(v^{*} v_{\xi}-v v_{\xi}^{*}+u^{*} u_{\xi}-u u_{\xi}^{*}\right) v+q_{3}\left(|v|^{4}+6|v|^{2}|u|^{2}+3|u|^{4}\right) v=0,
\end{aligned}
$$

where $q_{1}, q_{2}$ and $q_{3}$ are constants, and look for some special solutions in the form of travelling waves (travelling wave solutions to (33) can be found similarly). We use a modified form of the so-called tangent hyperbolic method in order to find sech, tanh or mixed-type solutions to the coupled system (35).

In recent years, various methods have been proposed for obtaining explicit travelling solitary wave solutions to nonlinear evolution equations. The mixing exponential method developed by Hereman and Takaoka [20] and the so-called hyperbolic tangent method developed by Malfiert [21] may be mentioned among the exact methods. The basic idea in the tanh method is to express a solution as a polynomial in terms of a tanh function in which the degree of the polynomial is determined by a balancing procedure. Substituting the solution into the evolution equation leads to a set of algebraic equations. The solution of the set provides a solution to the evolution equation. In recent years, various generalizations of this tanh method have been presented for finding multiple travelling wave solutions to nonlinear evolution equations. According to the method presented in [12], nonlinear partial differential equations

$$
P\left(u, v, u_{t}, v_{t}, u_{x}, v_{x}, \ldots\right)=0, \quad Q\left(u, v, u_{t}, v_{t}, u_{x}, v_{x}, \ldots\right)=0
$$

are transformed into nonlinear ordinary differential equations through a travelling wave transformation $\zeta=x+\lambda t$ :

$$
P\left(U, V, U^{\prime}, V^{\prime}, \ldots\right)=0, \quad Q\left(U, V, U^{\prime}, V^{\prime}, \ldots\right)=0
$$

where $u=U(\zeta), v=V(\zeta)$, and ' denotes the partial differentiation with respect to $\zeta$. Then, new variables $\varphi=\varphi(\zeta), \psi=\psi(\zeta)$ which are solutions of the coupled Riccati equations are introduced

$$
\psi^{\prime}=-k \psi \varphi, \quad \varphi^{\prime}=k\left(1-\varphi^{2}\right) .
$$

A solution of (37) is then expressed as a sum of finite series

$U(\zeta)=\sum_{i=0}^{m} a_{i} \psi^{i}+\sum_{i=1}^{m} b_{i} \varphi \psi^{i-1}, \quad V(\zeta)=\sum_{j=0}^{n} c_{j} \psi^{j}+\sum_{j=1}^{n} d_{j} \varphi \psi^{j-1}$

where $a_{i}, b_{i}, c_{j}$ and $d_{j}$ are constants. Moreover, positive integers $m$ and $n$ are determined by balancing the highest derivative term with the highest nonlinear term. Then substituting solution (39) into the system and solving the resulting nonlinear algebraic system gives multiple travelling wave solutions of the nonlinear partial differential equation (36). This method is 
applied to various nonlinear evolution equations to obtain travelling wave solutions. All the evolution equations considered in [12] are quadratically nonlinear, and consequently no problem appears in balancing dispersive and nonlinear terms. However, if the nonlinearity is of fifth order as in the case of the CQNLS equations, it is impossible to balance the dispersive term and the nonlinear term. In other words, $m$ and $n$ are obtained as rational numbers if $\varphi$ and $\psi$ are solutions of the coupled Riccati equations (40), that is the method does not work successfully. In order to overcome this difficulty we assume the new variables $\varphi$ and $\psi$ are solutions of the system

$$
\varphi^{\prime}=k \varphi\left(1-\frac{1}{2} \varphi^{2}\right), \quad \psi^{\prime}=\frac{k}{2} \psi\left(1-\varphi^{2}\right) .
$$

Explicit tanh-type and sech-type solutions of system (40) are given by

$$
\varphi^{2}(\zeta)=1+\tanh k \zeta, \quad \psi^{2}(\zeta)=\operatorname{sech} k \zeta,
$$

where $\psi^{4}=1-\left(\varphi^{2}-1\right)^{2}$. A solution of the form (39) is then assumed. Substituting the solution into the CQNLS system leads to a system of nonlinear algebraic equations. If we can find a real non-trivial solution to these algebraic equations, a modified form of the tanh method based on utilizing combinations of solutions to the coupled ordinary differential equations (40) works successfully, at least for the CQNLS system.

In order to find travelling waves of equations (35), we introduce the travelling wave transformation

$u(\xi, \tau)=U(\zeta) \exp \left\{\mathrm{i}\left[k \xi-q_{1}\left(k^{2}+\alpha\right) \tau\right]\right\}, \quad v(\xi, \tau)=V(\zeta) \exp \left\{\mathrm{i}\left[k \xi-q_{1}\left(k^{2}+\alpha\right) \tau\right]\right\}$,

where $k$ and $\alpha$ are arbitrary parameters and $\zeta=\xi-2 k q_{1} \tau$. Substituting solutions (42) into system (35) leads to a pair of coupled ordinary nonlinear differential equations for the real amplitudes $U$ and $V$

$$
\begin{aligned}
& U^{\prime \prime}+\alpha U+\gamma\left(U^{2}+V^{2}\right) U+\beta\left(U^{4}+6 U^{2} V^{2}+3 V^{4}\right) U=0, \\
& V^{\prime \prime}+\alpha V+\gamma\left(V^{2}+U^{2}\right) V+\beta\left(V^{4}+6 U^{2} V^{2}+3 U^{4}\right) V=0,
\end{aligned}
$$

where prime denotes the differentiation with respect to $\zeta$, and the coefficients are defined as $\gamma=-2 k q_{2} / q_{1}, \beta=q_{3} / q_{1}$.

We now assume that system (43) has solutions of the form (39). Then, positive integers $m$ and $n$ are determined by balancing the dispersive terms $U^{\prime \prime}$ with the highest nonlinear terms such as $U^{5}, U^{3} V^{2}, \ldots$ in the differential equation system (43). Using solutions (39) and coupled differential equations for $\varphi$ and $\psi(40)$, we obtain $m=n=1$, and hence

$$
U=a_{0}+a_{1} \psi+a_{2} \varphi, \quad V=b_{0}+b_{1} \psi+b_{2} \varphi .
$$

Substituting solutions (44) into (43) and replacing derivative terms by polynomial expressions in $\psi$ and $\varphi$, we obtain two algebraic equations in mixed powers of $\varphi$ and $\psi$. Because of the relation $\psi^{4}=1-\left(\varphi^{2}-1\right)^{2}$, we can express the term $\psi^{4}$ as polynomials in $\varphi$ in the resulting equations. Equating the coefficient of each power of the variables $\varphi$ and $\psi$ leads to a nonlinear system of algebraic equations involving the parameters $a_{i}$ and $b_{i},(i=0,1,2)$. Inspection of the resulting nonlinear algebraic equations shows that system (43) has only sech type, i.e. $\psi(\zeta)$, or tanh type, i.e. $\varphi(\zeta)$, solutions. On the basis of this observation, we obtain the following special solutions to system (43).

Case $A$. In order to simplify the nonlinear algebraic equation to be solved, we have assumed that $a_{0}=0, a_{2}=0, b_{0}=0$ and $b_{2}=0$ for the sech-type solutions. Then, the following set of 

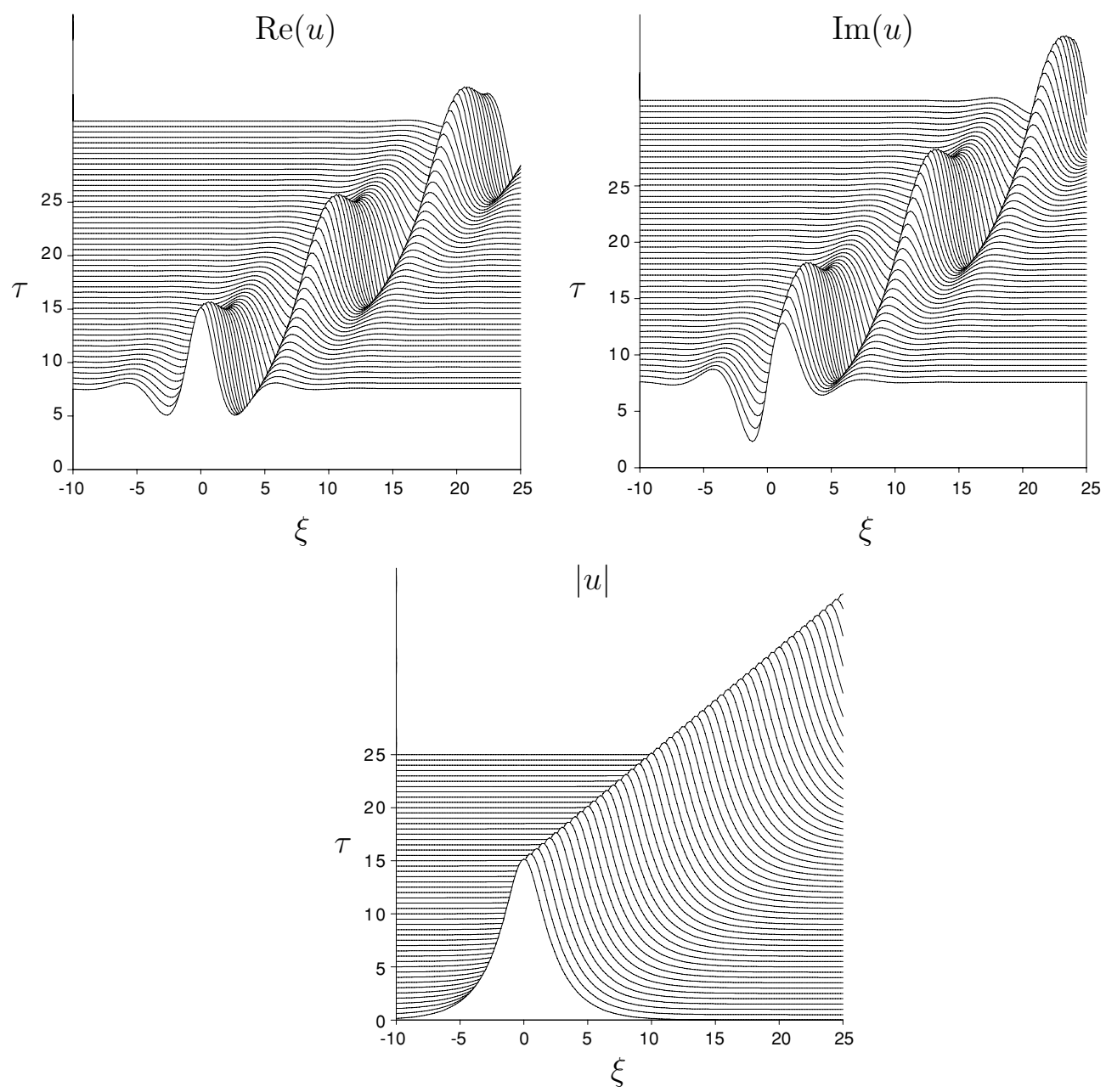

Figure 1. 3D plot of the real and imaginary parts and the modulus of the sech-type solution versus $\xi$ and $\tau$ for $q_{1}=1 / 2, q_{2}=0$ and $q_{3}=20 / 3$.

equations for the parameters $a_{1}, b_{1}, k, \alpha$ and $\beta$ is obtained:

$$
\begin{array}{lll}
\psi: & a_{1}\left(k^{2}+4 \alpha\right)=0, & b_{1}\left(k^{2}+4 \alpha\right)=0, \\
\varphi^{2} \psi: & a_{1}\left[-3 k^{2}+4 \beta\left(a_{1}^{4}+6 a_{1}^{2} b_{1}^{2}+3 b_{1}^{4}\right)\right]=0, & b_{1}\left[-3 k^{2}+4 \beta\left(b_{1}^{4}+6 a_{1}^{2} b_{1}^{2}+3 a_{1}^{4}\right)\right]=0, \\
\psi^{3}: & a_{1} \gamma\left(a_{1}^{2}+b_{1}^{2}\right)=0, & b_{1} \gamma\left(a_{1}^{2}+b_{1}^{2}\right)=0, \\
\varphi^{4} \psi: & a_{1}\left[3 k^{2}-4 \beta\left(a_{1}^{4}+6 a_{1}^{2} b_{1}^{2}+3 b_{1}^{4}\right)\right]=0, & b_{1}\left[3 k^{2}-4 \beta\left(b_{1}^{4}+6 a_{1}^{2} b_{1}^{2}+3 a_{1}^{4}\right)\right]=0 .
\end{array}
$$

Solving the nonlinear system of equations (45) gives a set of admissible solutions:

$$
U(\zeta)=a_{1} \sqrt{\operatorname{sech} k \zeta}, \quad V(\zeta)= \pm a_{1} \sqrt{\operatorname{sech} k \zeta}
$$

where

$$
\alpha=-\frac{k^{2}}{4}, \quad a_{1}^{2}=b_{1}^{2}=\frac{\sqrt{3 k^{2}}}{2 \sqrt{10 \beta}},
$$




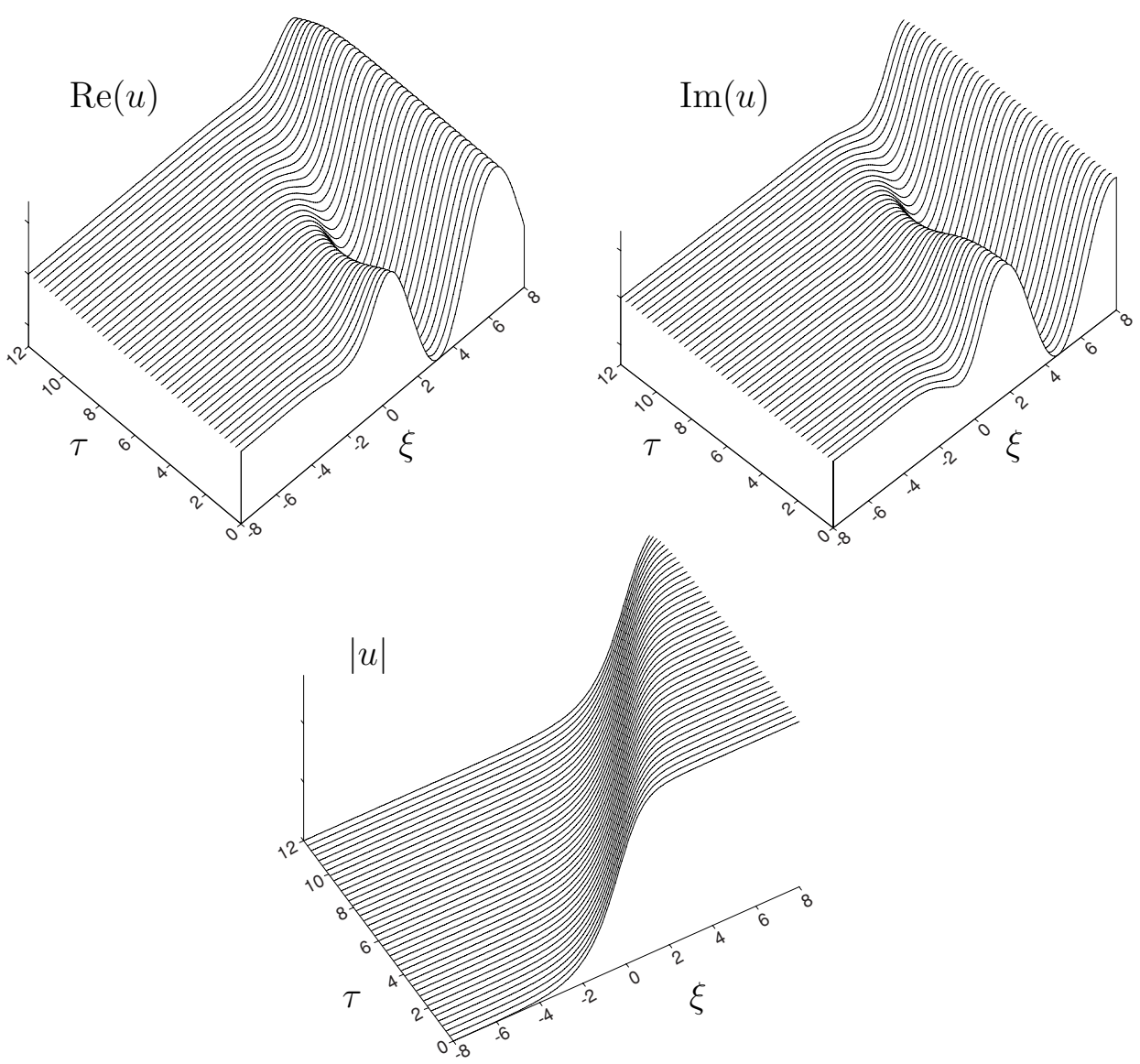

Figure 2. 3D plot of the real and imaginary parts and the modulus of the tanh-type solution versus $\xi$ and $\tau$ for $q_{1}=3 / 10, q_{2}=-3 / 20$ and $q_{3}=-9 / 400$.

with $\gamma=0, \alpha<0$ and $\beta>0$. In order to obtain a non-trivial solution for this particular case, the coefficient $\gamma$ is assumed to be zero. Therefore, the set (46) is a solution to the pure quintic system where cubic nonlinear effects are ignored:

$$
\begin{aligned}
& U^{\prime \prime}+\alpha U+\beta\left(U^{4}+6 U^{2} V^{2}+3 V^{4}\right) U=0, \\
& V^{\prime \prime}+\alpha V+\beta\left(V^{4}+6 U^{2} V^{2}+3 U^{4}\right) V=0 .
\end{aligned}
$$

In fact, the sech-type solution (46) of the CQNLS system is also a travelling wave solution of a single QNLS equation $U^{\prime \prime}+\alpha U+10 \beta U^{5}=0$ because $U= \pm V$. This restricted solution to the CQNLS system is basically due to a symmetrical coupled structure of the system. A three-dimensional plot of the solution (42) corresponding to this particular case is shown in figure 1. As a final comment to this subsection, one could mention that the bright envelope solitary wave solution of the cubic NLS equation has the form $u=\operatorname{sech} \zeta \mathrm{e}^{\mathrm{i} \theta}$ where the coefficients are omitted. This results from the balance between the cubic nonlinearity and the dispersion. On the other hand, the perfect balance between the quintic nonlinearity and the dispersion gives a solution of the form $u=\sqrt{\operatorname{sech} \zeta} \mathrm{e}^{\mathrm{i} \theta}$ to the quintic NLS equation.

Case $B$. In order to find tanh-type solutions, we assume that $a_{0}=0, a_{1}=0, b_{0}=0$ and $b_{1}=0$. Then, the following set of equations for the parameters $a_{2}, b_{2}, k, \alpha$ and $\beta$ is obtained: 


$$
\begin{array}{lll}
\varphi: & a_{2}\left(k^{2}+\alpha\right)=0, & b_{2}\left(k^{2}+\alpha\right)=0, \\
\varphi^{3}: & a_{2}\left[-2 k^{2}+\gamma\left(a_{2}^{2}+b_{2}^{2}\right)\right]=0, & b_{2}\left[-2 k^{2}+\gamma\left(a_{2}^{2}+b_{2}^{2}\right)\right]=0, \\
\varphi^{5}: & a_{2}\left[3 k^{2}+4 \beta\left(a_{2}^{4}+6 a_{2}^{2} b_{2}^{2}+3 b_{2}^{4}\right)\right]=0, & b_{2}\left[3 k^{2}+4 \beta\left(b_{2}^{4}+6 a_{2}^{2} b_{2}^{2}+3 a_{2}^{4}\right)\right]=0 .
\end{array}
$$

Solving the nonlinear system of equations (48) gives a set of admissible solutions:

$$
U(\zeta)=a_{2} \sqrt{1+\tanh k \zeta}, \quad V(\zeta)= \pm a_{2} \sqrt{1+\tanh k \zeta}
$$

where

$$
\alpha=-k^{2}, \quad a_{2}^{2}=b_{2}^{2}=\frac{k^{2}}{\gamma}, \quad \beta=-\frac{3 \gamma^{2}}{40 k^{2}}
$$

with $\gamma>0, \alpha<0$ and $\beta<0$. Unlike the previous case, set (49) is a solution to the coupled system (43) where both cubic and quintic nonlinear effects are present. However, similar to the previous case, the tanh-type solution (49) of the CQNLS system is also a travelling wave solution of a single QNLS equation $U^{\prime \prime}+\alpha U+2 \gamma U^{3}+10 \beta U^{5}=0$ because $U= \pm V$. A three-dimensional plot of solution (42) corresponding to this particular case is shown in figure 2. Similar to the previous case, the envelope solitary wave solution is a result of the quintic nonlinearity and the dispersion.

We should also note that we could not obtain a non-trivial solution to the nonlinear algebraic equations if we assume mixed-type solutions of the form (44). This is due to the fact that different restrictions are obtained for the sech-type solutions, i.e. $\gamma=0$ and $\beta>0$, and for the tanh-type solutions, i.e. $\gamma>0$ and $\beta<0$.

\section{Conclusions}

As is well known, nonlinear modulation of one-dimensional waves on the surface of water is described by the cubic NLS equation [1]. When the coefficient of the nonlinear term of the cubic NLS equation is zero for the value of $k h=1.363$, the NLS equation is not valid and, as mentioned in the introduction, a quintic nonlinear Schrödinger equation has been obtained for waves on the surface of water in [3, 4] near the critical wavenumber. The specific number in the case of water waves does not depend on any parameter characterizing the medium. However, this is not the case for an elastic medium where coefficients of nonlinear terms in NLS equation usually depend on material parameters as well as wavenumber. Therefore, the existence of such a critical wavenumber for waves propagating in a bulk elastic medium will imply a condition similar to that of (13), between the elastic constants. In the present study, a system of two coupled quintic NLS equations is derived for two transverse waves propagating in a bulk elastic medium, under the assumption of the existence of a critical wavenumber. Whether such an exceptional elastic material exists depends on the knowledge of linear, second-order and third-order elastic constants. The values of linear and second-order elastic constants for a number of materials are given in [14]. Since the values of third-order elastic constants are not listed in [14], we are not able to check a possibility for the existence of such an elastic material. However, results presented in this study could possibly be significant for wave phenomena in various continuous media, including optical effects. As an example, we could mention that the interaction of optical solitons in two-mode fibres and birefringent fibres is governed by a pair of coupled NLS equations. If a critical wavenumber exists in such optical phenomena, the results of the present study could be extended to study pulse propagation through optical fibres. Pulse propagation can be significant for practical applications in the area of optical fibre communication systems. 


\section{Acknowledgment}

The authors thank the referees for their useful and valuable suggestions.

\section{References}

[1] Hasimoto H and Ono H 1972 J. Phys. Soc. Japan 33 805-11

[2] Hasegawa A and Tappert F 1973 Appl. Phys. Lett. 23 142-4, 171-2

[3] Johnson R S 1977 Proc. R. Soc. A 357 131-41

[4] Kakutani T and Michihiro K 1983 J. Phys. Soc. Japan 52 4129-37

[5] Parkes E J 1987 J. Phys. A: Math. Gen. 20 2025-36

[6] Erbay S 1995 J. Phys. A: Math. Gen. 28 2905-19

[7] Kundu A 1987 Physica D 25 399-406

[8] Pathria D and Morris J Ll 1989 Phys. Scr. 39 673-9

[9] Clarkson P A 1992 Nonlinearity 5 453-72

[10] Newboult G K, Parker D F and Faulkner T R 1989 J. Math. Phys. 30 930-6

[11] Erbay H A, Erbay S and Dost S 1991 Int. J. Eng. Sci. 29 859-68

[12] Yao R X and Li Z B 2002 Phys. Lett. A 297 196-204

[13] Suhubi E S and Eringen A C 1964 Int. J. Eng. Sci. 2 389-404

[14] Erofeyev V I and Potapov A I 1993 Int. J. Non-Linear Mech. 28 483-8

[15] Maugin G A, Hadouaj H and Malomed B A 1992 Phys. Rev. B 45 9688-94

[16] Maugin G A 1985 Nonlinear Electromechanical Effects and Applications (Singapore: World Scientific)

[17] Maugin G A 1999 Nonlinear Waves in Elastic Crystals (New York: Oxford University Press)

[18] Taniuiti T 1974 Prog. Theor. Phys. Suppl. 55 1-35

[19] Hacinliyan I and Erbay S 2002 Chaos Solitons Fractals 14 1127-35

[20] Hereman W and Takaoka M 1990 J. Phys. A: Math. Gen. 23 4805-22

[21] Malfiert W 1992 Am. J. Phys. 60 650-54

[22] Fedele R 2002 Phys. Scr. 65 502-8 